\title{
Better Understanding of SN Ia from Near Infrared Observations
}

\author{
Robert P. Kirshner \\ Harvard-Smithsonian Center for Astrophysics \\ 60 Garden Street, Cambridge, MA 02138, USA
}

\begin{abstract}
Type Ia supernovae (SN Ia) are explosions of white dwarfs whose distances can be measured to a precision of $\sim 5 \%$ using luminosity information that is encoded in the light curve shape. This property has been very successfully exploited to measure the history of cosmic expansion and to infer the presence of dark energy. But to learn the properties of dark energy and determine whether it is different from the cosmological constant demands higher precision and better accuracy than optical light curves alone can provide. The largest systematic uncertainties come from light curve fitters, photometric calibration errors, and from poor knowledge of the scattering properties of dust along the line of sight. Efforts to use SN Ia spectra as luminosity indicators have had some success, but have not produced a big step forward. Fortunately, observations of SN Ia in the near infrared (NIR), from 1 to 2 microns, offer a very promising path to better knowledge of the Hubble constant, improved constraints on dark energy, and, possibly, a route to discriminating the progenitor paths for SN Ia explosions.
\end{abstract}

Keywords. supernovae: general - cosmology: distance scale, cosmological parameters

\section{Type Ia Supernovae as Exploding White Dwarfs}

At the dawn of cosmological time, in 1921, Harlow Shapley noted that if the bright novae in spiral nebulae were the same as novae in the Milky Way, the spirals must be local. As part of his argument that the nebulae were not part of the Milky Way, Heber D. Curtis lamely countered, "a division into two classes" of novae "is not impossible" (Trimble 1995). Once Hubble (1925) demonstrated that the spiral nebulae were, in fact, extragalactic, the need for super-novae was clear, at least to Fritz Zwicky who speculated, along with Walter Baade, that the origin of their energy could be the gravitational collapse to a neutron star (Baade \& Zwicky 1934). This, of course, is completely wrong for the supernovae they observed through the 1930s, which were all of the thermonuclear type we now call Type Ia. Despite this profound lack of understanding, Baade (1938) showed they were pretty good standard candles suitable for cosmological distance measurement.

The perfection of this technique, barging ahead without full understanding, has been a long and glorious one leading to the Nobel Prize in Physics in 2011 to Saul Perlmutter, Brian Schmidt, and Adam Riess "for the discovery of the accelerating expansion of the Universe through observations of distant supernovae." This is evidence that we can make progress in astronomy even without correct understanding (Kirshner 2009). But it would be much better, both intellectually and practically, if we knew what we were talking about.

The modern picture of Type Ia supernovae as exploding white dwarfs dates at least from 1960, when Fowler and Hoyle (1960) pointed out that stellar evolution branches at carbon ignition. Massive stars ignite carbon gently in their cores as a gas, while for low mass stars carbon ignition takes place violently in a degenerate core. The physical setting for degenerate carbon ignition is in a white dwarf. Two astronomical pictures that could 
lead to ignition of a white dwarf in a binary are mass accreted from a non-degenerate neighbor or the merger of two white dwarfs. This conference is a testament to the fact that we have not yet fully sorted out which of these paths is responsible for the events we detect and use to measure the Universe.

Even so, we are reasonably confident that the general picture is correct. Type Ia supernovae are found in all types of galaxies, including elliptical galaxies, where the current rate of star formation is low, so at least some of them must have a long fuse, which could come from the long path of binary evolution. The chemical abundances and velocity distribution of nuclear burning products in models of exploding white dwarfs match the spectra we observe near maximum light (Blondin et al. 2011) The nuclear physics of carbon burning naturally produces ${ }^{56} \mathrm{Ni}$, whose decay scheme through cobalt and iron matches the energetics of SN Ia, the evolving atmospheric abundances of cobalt and iron (Kuchner et al. 1994), and the nebular spectrum observed at very late stages which is mostly due to iron emission lines (Kirshner \& Oke 1975). More directly, the very early light curves of SN 2011 fe imply the radius of the progenitor must have been small pointing to the origin of this event in the explosion of a white dwarf (Nugent et al. 2011.)

\section{Measuring Distances with SN Ia}

Following Baade's lead, many investigators have used SN Ia as standard candles to measure extragalactic distances from their flux. A good summary of the prehistoric work comes from Kowal (1968) who prophesied, "These supernovae could be exceedingly useful indicators of distance. It should be possible to obtain average supernova magnitudes to an accuracy of $5 \%$ to $10 \%$ in the distances." He also predicted the future use of supernovae to determine cosmic acceleration: "It may even be possible to determine the second-order term in the redshift-magnitude relation when light curves become available for very distant supernovae." The "second-order term" would be the one that indicates cosmic acceleration or deceleration.

Modern work with linear detectors has made this dream come true. But it is not quite as simple as Kowal thought.

First, SN Ib had to be clearly identified and removed from the SN Ia samples. We now understand that SN Ib (and their more extreme cousins, SN Ic) are completely different physical events, ascribed to core collapse in massive stars that have lost their hydrogen envelopes in late stages of stellar evolution (Uomoto \& Kirshner 1985, Schlegel \& Kirshner 1989, Filippenko 1997).

Next, the overly-simple assumption that SN Ia are standard candles had to be revised in the face of clear evidence of low-luminosity SN Ia like SN 1991bg (Leibundgut et al. 1993, Filippenko et al. 1992) and high-luminosity objects like SN1991T (Phillips et al. 1992). Fortunately, Mark Phillips (1993) was able to use the high-quality photometry gathered at CTIO to see that there was a relation between the light curve shape and the luminosity for SN Ia. The B and V band light curves in the weeks around maximum light establish both the luminosity and the reddening to SN Ia (Phillips et al. 1999, Riess, Press, \& Kirshner 1996a.) The scatter about the Hubble line for well-observed supernovae in nearby galaxies can be reduced to about $10 \%$ in distance by these and related methods (Figure 1).

Riess, Press, \& Kirshner (1996b) tried to see if the dust absorbing the light of supernovae in other galaxies was the same as dust in the Milky Way. In their small sample, this appeared to be true, but more recent work has shown that some supernova results favor a value of $R_{V}$ as small as 1.7 (Goobar 2008). 


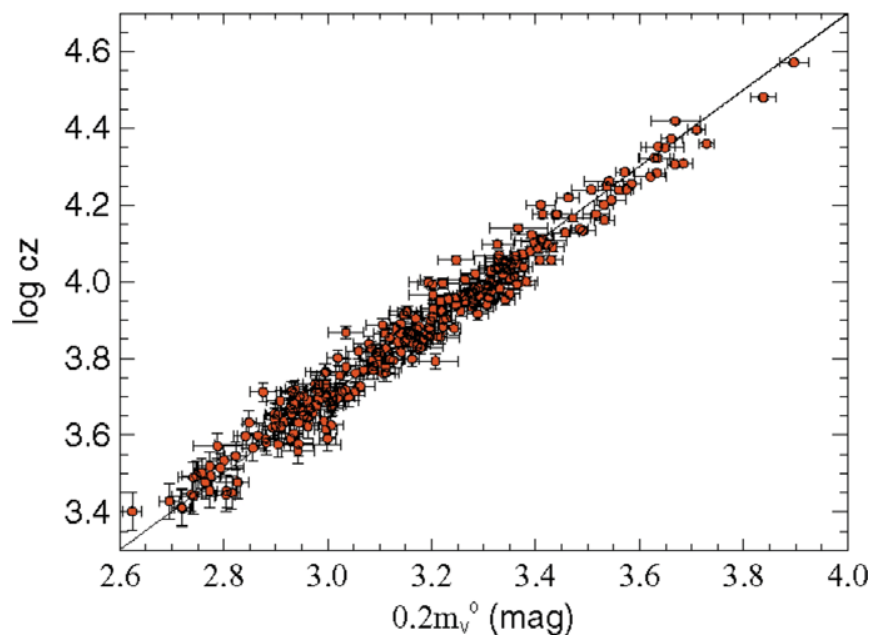

Figure 1. SN Ia Hubble diagram based on the CfA3 sample of Hicken et al. (2009). The small scatter is the result of good photometry plus the methods for using light curve shape to determine the true luminosity.

Dust absorption is potentially important in making cosmological measurements: the dimming at redshift 0.5 due to cosmological acceleration is about $0.2 \mathrm{mag}$, which could be produced by dust (if its like Milky Way dust) that changes the B-V color by only about 0.07 mag. Accurate photometry in multiple bands was essential to make reliable inferences about cosmology. The earliest work by the Supernova Cosmology Project (SCP) did not take account of this - the first measurements were made in only one filter (Perlmutter et al. 1995) and the first cosmological results (Perlmutter et al. 1997) seemed to point toward a high-density universe that they said was "inconsistent with Lambda-dominated, low-density, flat cosmologies." This view was soon revised by publication in 1998 of results from the High-Z Team (HZT, Garnavich 1998) and by the SCP (Perlmutter et al. 1998) that showed no evidence for deceleration from a high-density universe, and then the decisive results from the HZT in 1998 (Riess et al. 1998) and from the SCP in 1999 (Perlmutter et al. 1999) with clear evidence for cosmic acceleration.

Since 1998, the case for cosmic acceleration has grown stronger due to increased samples of SN Ia at all redshifts. These worldwide efforts have been summarized by Amanullah (2010) and by Suzuki (2011). As the samples have grown, the error ellipse on the presence of dark energy has shrunk, and now the conclusion of cosmic acceleration is firm enough to convince even the conservative Swedes of the Nobel Prize committee.

The conventional way to analyze the results also aims to constrain the equation-ofstate parameter, $w=p / \rho$, for dark energy. Lemaître explained (1934) that the vacuum energy of the cosmological constant would act like a negative pressure in which $p=$ $-\rho c^{2}$ : in the modern language, we'd call that an equation of state value of $w=-1$. Suzuki's analysis of the world's data shows that our current measurements of the quantity $(1+w)=-0.013 \pm 0.07$ with a systematic error that is bigger than the statistical one. In other words, observations of SN Ia are consistent with the dark energy being the cosmological constant, but the systematic errors, which stem from the uncertainties in light curve fitters, photometric calibration, and in dust properties, are larger than the random ones. We will not make good progress constraining dark energy with supernovae without making progress on these sources of systematic error.

We've come a long way without a proper understanding of the astronomical origin of thermonuclear supernova explosions or the properties of the dust that obscures them. 
This was good enough when the goal was to establish the reality of cosmic acceleration. Now we want to constrain the nature of the dark energy. We need to do better.

\section{Clues from Spectra?}

As radiation escapes from the atmosphere of an SN Ia, it diffuses down a velocity gradient that embeds a complex three-dimensional mixture of elemental abundances set up by the nuclear flame that ripped through the degenerate star. The spectrum of an SN Ia, properly read, contains clues to the nature of the explosion that could supplement the information in the light curve to improve distance estimates (Blondin et al. 2012). It also seems quite plausible that the spectrum might also reveal signatures that could distinguish the explosion of one white dwarf from the merger of two.

One recent comparison of SN Ia models with observed spectra has been carried out by Blondin et al. (2011). The models come from the survey of 44 two-dimensional delayeddetonation models computed by Kasen et al. (2009), each viewed from multiple directions. The data come from the CfA, and include optical light curves of 251 SN Ia and 2231 low-dispersion spectra of the same objects. Kasen et al. had already shown that their models span the range of SN Ia luminosities and light curve shapes, and reproduce the observed relation between those two quantities. But it is important to show that the same models that reproduce high-luminosity, slowly-declining supernovae or lowluminosity, fast-declining supernovae also have spectra that look like supernovae that nature produces. These models have high enough fidelity that we were able to use the same tools developed for the observations to analyze the light curves and the SuperNova IDentification (SNID) code of Blondin \& Tonry (2007) for the spectra. The ability to use tools developed for observational data on the models marks a significant step forward in the realism of the models. Blondin et al. showed that the models that match observed spectra best lie on the observed width-luminosity relation. Conversely, we rejected six models with highly asymmetric ignition conditions and a large amount $\left(\geqslant 1 \mathrm{M}_{\odot}\right)$ of synthesized ${ }^{56} \mathrm{Ni}$ because they yield poor matches to observed SN Ia spectra. Theorists are able to construct models that nature does not produce in real explosions, and observers can now rule these out from combining light curves and spectra. This is progress!

Another recent paper using the CfA sample but more sharply focused on the question of improving distances to supernovae is by Blondin, Mandel, \& Kirshner (2011). Following the idea introduced by Bailey et al. (2009), we explored the flux ratios within a spectrum that are correlated with the supernova luminosity. The best of these produces a $30 \%$ improvement in the scatter of the Hubble diagram for this sample. We concluded that spectra do improve distance measurements of SN Ia, "but not as much as we hoped." I remain uncertain whether the extra effort required to measure a series of spectra for a cosmological sample would be repaid with better knowledge of dark energy. A similar investigation of the Berkeley KAIT spectra and light curves by Silverman et al. (2012) found similar results, but cast them in a more optimistic light.

Another thread of spectroscopic indicators has emphasized the ultraviolet spectra of SN Ia (Maguire et al. 2012). They conclude that the correlations of spectra with redshift and galaxy host properties are more useful in detecting the effect of cosmic evolution on progenitors than for improving SN Ia as cosmological probes. A different conclusion has been drawn by Foley (2012) and by Foley \& Kasen (2011) who noted a relation between ejecta velocity and intrinsic color that they say can improve the precision of SN Ia distances by a factor of two. It will be interesting to see if these trends can be harnessed as independent inputs to statistical models that predict supernova distances, like the BayeSN models developed by Mandel et al. (2009). 


\section{SN Ia in the NIR}

The most promising development for improving SN Ia distances has come from observations made at near-infrared wavelengths (1 to 2 microns: $\mathrm{y}, \mathrm{J}, \mathrm{H}, \mathrm{K}$ bands.) The pioneering work by Krisciunas et al. (2007) and subsequent observations by the Carnegie group (Folatelli et al. 2010) have been augmented by an ongoing program at the CfA that exploits the PAIRITEL $1.3 \mathrm{~m}$ at Mount Hopkins, Arizona. This is the former 2MASS telescope with a 2MASS camera that was converted to a robotic system by Josh Bloom to study gamma-ray bursts. Since gamma-ray bursts are rare, the telescope is often available to monitor supernovae in the $\mathrm{J}, \mathrm{H}, \mathrm{K}_{\mathrm{s}}$ bands of the 2MASS system. A preliminary report by Wood-Vasey et al. (2008) gives NIR light curves for 21 SN Ia, and a much larger sample will be forthcoming in the Ph.D. thesis of Andrew Friedman (2012).

The dispersion in absolute magnitudes for these NIR bands is much smaller than the range observed at optical wavelengths (Figure 2). In fact, SN Ia in the NIR are better standard candles without any correction for light curve shape than optical observations of SN Ia after all the prestidigitation of light curve shape analysis (Mandel, Narayan, \& Kirshner 2011). A recent paper by Barone-Nugent et al. (2012) has similar results.

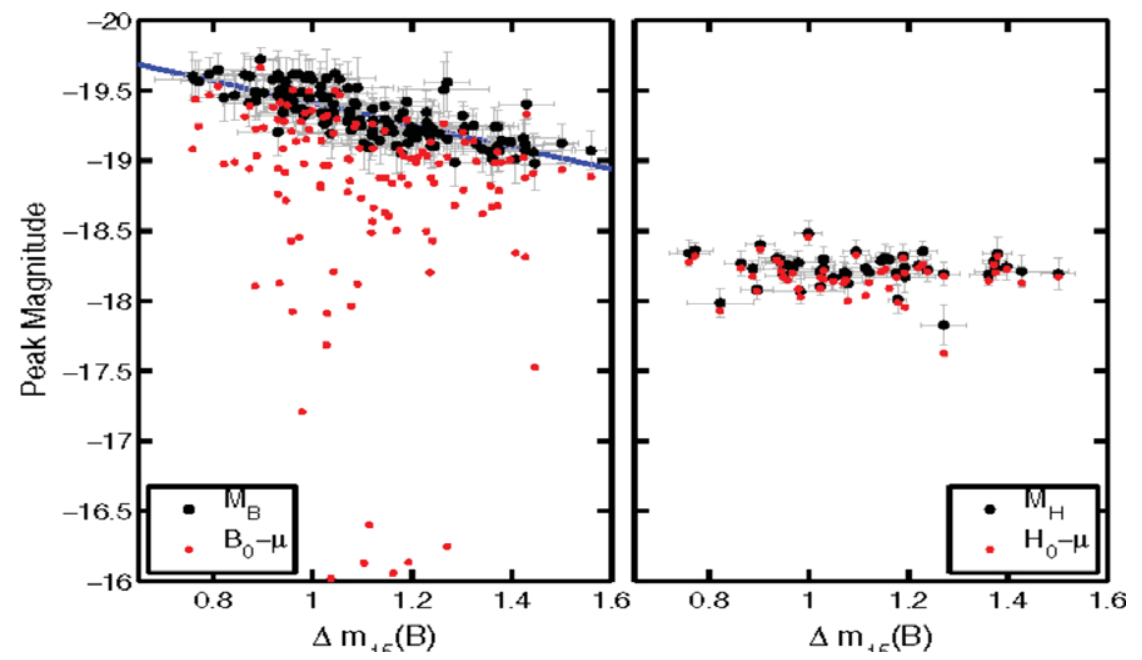

Figure 2. Luminosity-light curve shape relations in the optical (left) and the NIR (right) after Mandel, Narayan, \& Kirshner (2011). The solid blue line shows the usual relation between luminosity and light curve shape. The light, small, red dots are the observed points, while the large, black dots are for the same supernovae corrected for extinction. On the right, you can see that the relation between infrared luminosity and light curve shape is flat: the SN Ia are actually standard candles in the NIR. The corrections for dust absorption are also small.

This is not just an empirical fact, but was predicted by Dan Kasen in a 2006 paper (Kasen 2006). He considered the opacity effects of iron in the expanding atmosphere of SN Ia. The IR emission is produced by fluorescence from light absorbed in the UV by $\mathrm{Fe}+$ or $\mathrm{Fe}++$ that is re-emitted at the $\mathrm{J}, \mathrm{H}$, and $\mathrm{K}$ wavelengths. Kasen modeled the atmospheres of exploding white dwarfs with a range of energy inputs. He found that the corresponding range of light output was very large in the ultraviolet, but small in the NIR, and smallest at H-band (1.6 microns), just as observed. So SN Ia are better standard candles in the NIR. And we understand why!

It is also true that extinction, which scales roughly as $1 / \lambda$, is about 4 times smaller at $\mathrm{H}$-band than in the B-band. So even if there are some remaining uncertainties in the extinction and reddening properties of the dust obscuring supernovae, their effect is 
significantly smaller in the NIR (Figure 2). At the CfA, we have been using our $1.2 \mathrm{~m}$ telescope to obtain optical light curves of the same supernovae for which we gather the NIR data (Hicken et al. 2012). This wide span in wavelength makes it possible to estimate the amount of extinction $A_{V}$, and to infer the ratio of total to selective absorption, $R_{V}$. This analysis has been implemented in a hierarchical Bayesian framework dubbed BayeSN by Mandel et al. (2009).

The results are very gratifying: the prediction error for SN Ia distances shrinks when the IR data are included, and the insensitivity to extinction implies that the systematic errors should also be significantly reduced (Figure 3 ). It is even possible to show that the best value for $R_{V}$ is near the Milky Way value of 3.1 for low extinction cases, and only drifts into the peculiar zone of $\mathrm{R}_{\mathrm{V}} \sim 1.7$ for objects with high extinction. A similar result has been obtained by Chotard (2011). Whether this is connected to the multiplescattering idea of local dust suggested by Goobar (2008) remains to be established. This could be a hint of the circumstellar environment for some supernovae, and related to the life cycle of their progenitors.

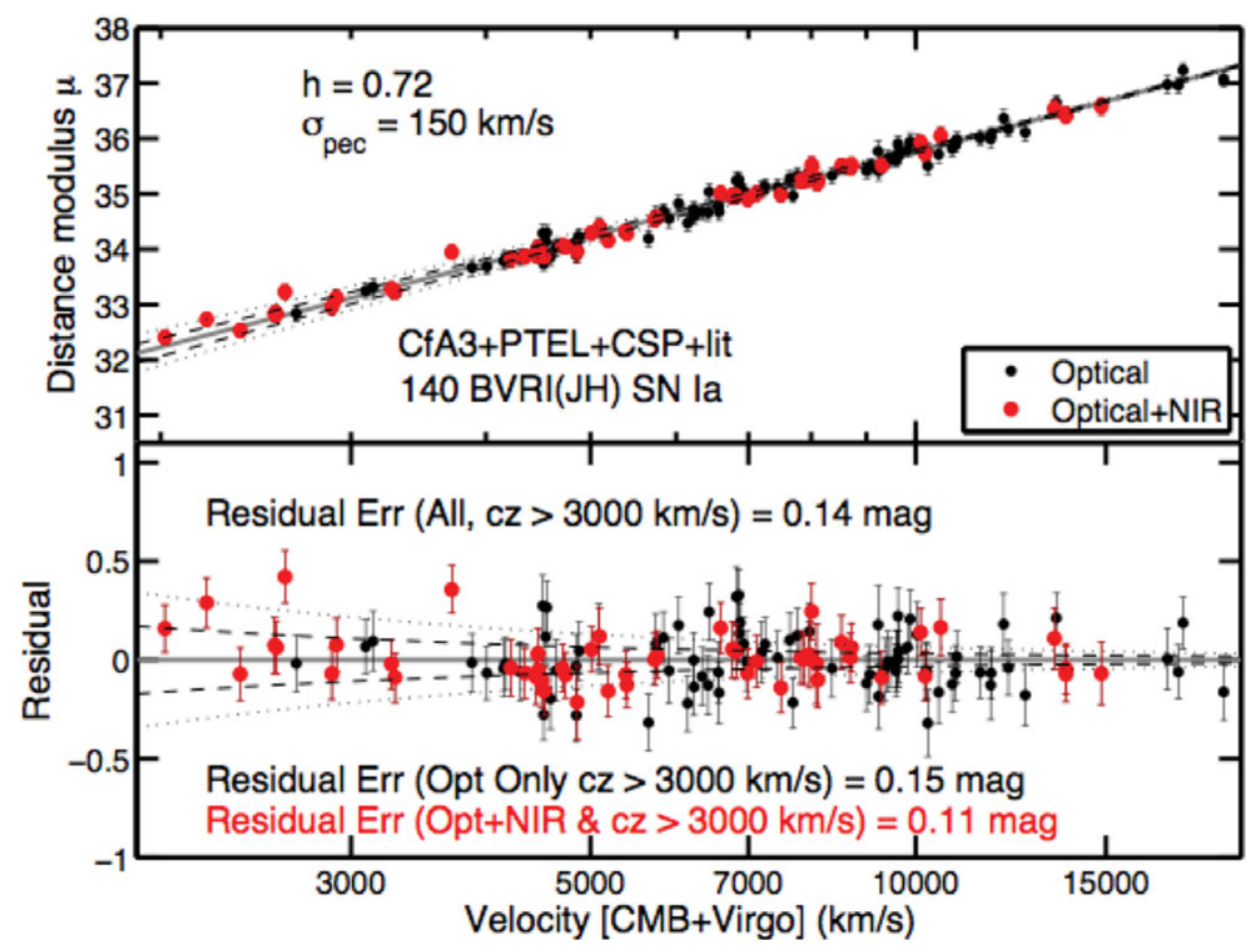

Figure 3. Improved low-z Hubble diagram using optical and NIR data after Mandel, Narayan, \& Kirshner (2011). The prediction error for distances is only $5 \%$.

The application of these BayeSN techniques to the full sample of Friedman's thesis is underway. This should provide further improvements in the low-redshift Hubble diagram, and could lead to higher precision in measuring the Hubble constant, $\mathrm{H}_{\mathrm{o}}$. This is of continued interest - for example the number of relativistic species (neutrinos) that are needed to model the CMB results seems to be 4 (not 3!) when you constrain $\mathrm{H}_{\mathrm{o}}$ with the best direct measurements (Suyu et al. 2012). Nobody should confuse this with the detection of a sterile neutrino, but it is an intriguing state of the present data. 
The use of SN Ia in the NIR can also be extended to measuring dark energy properties. For Cycle 20, we have been awarded 100 orbits of HST time to observe NIR points on the light curves of $25 \mathrm{SN}$ Ia at $\mathrm{z} \sim 0.4$ that will pop out of the medium-deep survey on PanSTARRS. Using the WFC3-IR channel, we should be able to improve on the opticalonly constraints on dark energy and avoid the nasty systematic effects of uncertain dust properties. We call this program "RAISIN" (an anagram of "SN IA (in the) IR") with SN Ia playing the role of raisins in the clichéd metaphor of the expanding universe (Figure 4).

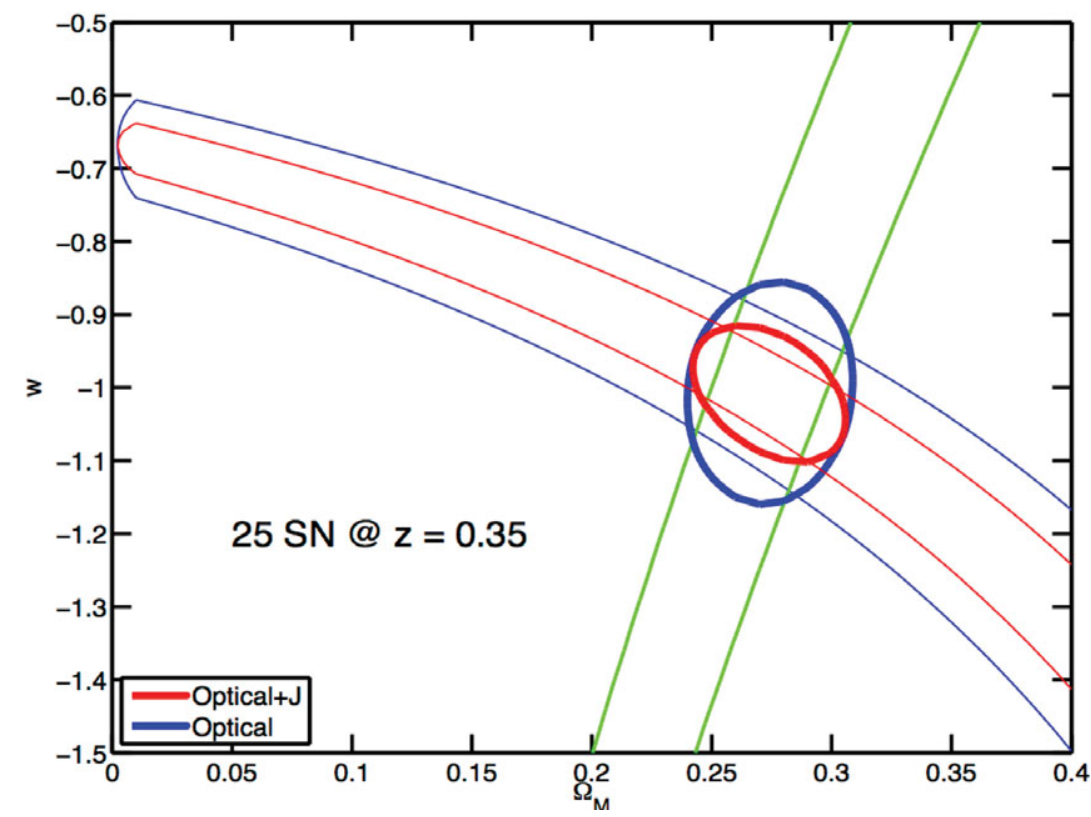

Figure 4. Expected improvement in constraints on dark energy properties due to RAISIN measurements with HST. The sample is only 25 objects, but should give a result which is statistically competitive with the world's sample of optical observations and less vulnerable to systematic errors from dust.

While this conference shows that there are deep uncertainties about the astronomical origin of SN Ia, their good behavior as cosmological distance indicators suggests either that different explosion mechanisms produce nearly identical light curves and spectra, or perhaps that most of the SN Ia we see come from a single mechanism, with a set of outliers that might represent another path to exploding a white dwarf. For example, the extra-bright SN Ia could be two white dwarfs merging while those with evidence for substantial circumstellar matter from absorption line studies (Dilday et al. 2012) might be good cases for one white dwarf with a mass-losing neighbor. The broad run of SN Ia of moderate luminosity seems likely to arise from a single mechanism with a range of energy inputs, rather than a superposition of two different types. But high-precision distances also mean very precise luminosity measurements, so the same work that aims at cosmological use of SN Ia may also help shed light on their astronomical origins.

\section{Acknowledgements}

Many thanks to my onetime postdoc Stéphane Blondin, at Centre de Physique des Particules in Marselles, and to recent Ph.D. students Malcolm Hicken, Kaisey Mandel, now at University College London, and Andy Friedman, now at MIT. Supernova work at Harvard is partially supported by NSF Grant AST 0907903. 


\section{References}

Amanullah, R. et al. 2010, ApJ, 716, 712

Baade, W. 1938, ApJ, 88, 285

Baade, W. \& Zwicky, F. 1934, PNAS, 20, 254

Bailey, S. et al. 2009, AछA, 500, L17

Barone-Nugent, R. L. et al. 2012, ArXiv, 1204.2308

Blondin, S. et al. 2011, MNRAS, 417, 1280

Blondin, S. \& Tonry, J. L. 2007, ApJ, 666, 1024

Blondin, S., Mandel, K., \& Kirshner, R. P. 2011, A\& $A$, 256, 81

Blondin, S. et al. 2012, AJ, 143, 126

Chotard, N. et al. 2011, A\&BA, 529, L4

Dilday, B. et al. 2012, ArXiv, 1207.1306

Filippenko, A. V. 1997, ARAA, 35, 309

Filippenko, A. V. et al. 1992, AJ, 104, 1543

Folatelli, G. et al. 2010, AJ, 139, 120

Foley, R. 2012, ArXiV, 1202.0003

Foley, R. \& Kasen, D. 2011, ApJ, 729, 55

Fowler, W. \& Hoyle, F. 1960, ApJ, 132, 565

Friedman, A. 2012, Ph.D. thesis, Harvard University

Garnavich, P. G. et al. 1998, ApJ, 493, L53

Goobar, A. 2008, ApJ, 686, L103

Hicken, M. et al. 2009, ApJ, 700, 331

Hicken, M. et al. 2012, ApJS, 200, 12

Hubble, E. 1925, ApJ, 62, 409

Kasen, D. 2006, ApJ, 649, 939

Kasen D., Röpke F. K., \& Woosley S. E., 2009, Nature, 460, 869

Kirshner, R. P. 2009, ArXiV, 0910.0257

Kirshner, R. P. \& Oke, J. B. 1975, ApJ, 200, 574

Kowal, C. T. 1968, AJ, 73, 1021

Krisciunas, K. et al. 2007, AJ, 133, 58

Kuchner, M., Kirshner, R. P., Pinto, P., \& Leibundgut, B. 1994, ApJ, 426, 89

Leibundgut, B. 1993, AJ, 105, 301

Lemaître, G. 1934, PNAS, 20, 12

Maguire et al. 2012, ArXiV, 1205.7040

Mandel, K. S. et al. 2009, ApJ, 704, 629

Mandel,K. S., Narayan, G. \& Kirshner, R. P. 2011, ApJ, 731, 120

Nugent, P. et al. 2011, Nature 480, 344

Perlmutter, S. et al. 1995, ApJ, 440, L41

Perlmutter, S. et al. 1997, ApJ, 483, 656

Perlmutter, S. et al. 1998, Nature, 391, 51

Perlmutter, S. et al. 1999, ApJ, 517, 565

Phillips, M. M. et al. 1992, AJ, 103, 163

Phillips, M. M. 1993, ApJ, 413, L10

Phillips, M. M. et al. 1999, AJ, 118, 1766

Riess, A. G., Press, W. H., \& Kirshner, R. P. 1996a, ApJ, 473, 88

Riess, A. G., Press, W. H., \& Kirshner, R. P. 1996b, ApJ, 473, 588

Riess, A. G. et al. 1998, AJ, 116, 1009

Schlegel, E. M. \& Kirshner, R. P. 1989, AJ, 98, 577

Silverman, J. et al. 2012, ArXiv, 1202.2130

Suyu, S. H. 2012, ArXiv, 1202.4459

Suzuki, N. et al. 2012, ApJ, 746, 85

Trimble, V. 1995, PASP, 107, 1133

Uomoto, A. \& Kirshner, R. P. 1985, A\& A, 149, 7

Wood-Vasey, W. M. et al. 2008, ApJ, 689, 377 